\title{
Calibration of the charge exchange recombination spectroscopy diagnostic for core poloidal rotation velocity measurements on JET
}

\author{
K. Crombée) \\ Department of Applied Physics, Ghent University, Rozier 44, B-9000, Ghent, Belgium \\ Y. Andrew, C. Giroud, and N. C. Hawkes \\ EURATOM/UKAEA Fusion Association, Culham Science Center, Abingdon OX14 3DB, United Kingdom \\ A. Murari and M. Valisa \\ Consorzio RFX, Associazione EURATOM-ENEA sulla Fusione, Corso Stat: Unit: 4 I-35127 Padova, Italy \\ G. Van Oost \\ Department of Applied Physics, Ghent University, Rozier 44, B-9000, Ghent, Belgium \\ K.-D. Zastrow and JET-EFDA Contributors \\ EURATOM/UKAEA Fusion Association, Culham Science Center, Abingdon OX14 3DB, United Kingdom
}

(Presented on 19 April 2004; published 1 October 2004)

This article describes recent improvements in the measurement of $\mathrm{C}^{6+}$ impurity ion poloidal rotation velocities in the core plasma of JET using charge exchange recombination spectroscopy. Two independent techniques are used to provide an accurate line calibration. The first method uses a Perkin-Elmer type 303-306 samarium hollow cathode discharge lamp, with a Sm I line at $528.291 \mathrm{~nm}$ close to the $\mathrm{C}$ vi line at $529.1 \mathrm{~nm}$. The second method uses the Be II at $527.06 \mathrm{~nm}$ and $\mathrm{C}$ III at $530.47 \mathrm{~nm}$ in the plasma spectrum as two marker lines on either side of the $\mathrm{C}$ VI line. Since the viewing chords have both a toroidal and poloidal component, it is important to determine the contribution of the toroidal rotation velocity component separately. The toroidal rotation velocity in the plasma core is measured with an independent charge exchange recombination spectroscopy diagnostic, looking tangentially at the plasma core. The contribution of this velocity along the lines of sight of the poloidal rotation diagnostic has been determined experimentally in $L$-mode plasmas keeping the poloidal component constant (K. Crombé et al., Proc. 30th EPS Conference, St. Petersburg, Russia, 7-11 July 2003, p. 1.55). The results from these experiments are compared with calculations of the toroidal contribution that take into account the original design parameters of the diagnostic and magnetic geometry of individual shots. () 2004 American Institute of Physics.

[DOI: $10.1063 / 1.1784531]$

\section{INTRODUCTION}

Systematic errors in the measurement of the core plasma impurity ion poloidal rotation velocity at JET $\left(v_{\text {pol }}\right)$ have been reduced by: (i) improving the determination of the line calibration and by (ii) quantifying the toroidal component along the lines of sight (LOS) of the diagnostic.

The diagnostic setup consists of six fibres providing $v_{\text {pol }}$ values at mid-plane radii between 3.30 and $3.70 \mathrm{~m}$, with a spatial resolution of $2-10 \mathrm{~cm}$ and a temporal resolution of $50 \mathrm{~ms}$. The spatial resolution is determined by the number of neutral injectors used and the specific magnetic configuration. A poloidal cross section of the diagnostic is shown schematically in Fig. 1 of Ref. 2. The wavelength calibration necessary for the Doppler spectroscopy velocity measurements is carried out using two separate techniques described in Sec. II. The viewing chords have a toroidal component with a magnitude of about $0.2-0.3 v_{\text {tor }}$ (with $v_{\text {tor }}$ the toroidal rotation velocity of the $\mathrm{C}^{6+}$ ion in the plasma core) and results from dedicated experiments to measure the toroidal

${ }^{a)}$ Electronic mail: k.crombe@fz-juelich.de contribution for each chord are reported in Sec. III. Corrections to the radial profiles due to temperature effects are discussed in Sec. IV, and radial $T_{i}$ profiles obtained with this charge exchange recombination spectroscopy (CXRS) diagnostic are compared with independent measurements of temperature in this region.

\section{CALIBRATION}

Two different calibration methods are used to obtain the unshifted wavelength of the $\mathrm{C}$ VI line $(529.1 \mathrm{~nm})$ as a reference for the Doppler shifted line. The dispersion of the instrument is determined using the spectrum from a PerkinElmer type 303-306 samarium hollow cathode discharge lamp, ${ }^{1}$ in the region $527.14-529.82 \mathrm{~nm}$, with four clearly identifiable lines. The lamp calibration is repeated each time a new series of measurements is taken. In addition, the unshifted wavelength position is monitored on a shot by shot basis using the plasma spectrum itself. The Be II line $(527.06 \mathrm{~nm})$ and the $\mathrm{C}$ III line $(530.47 \mathrm{~nm})$ are measured in the vicinity of the charge exchange $\mathrm{C}$ vI line. The $\mathrm{Be}^{1+}$ and $\mathrm{C}^{2+}$ ions are located in the scrape off layer, where plasma 


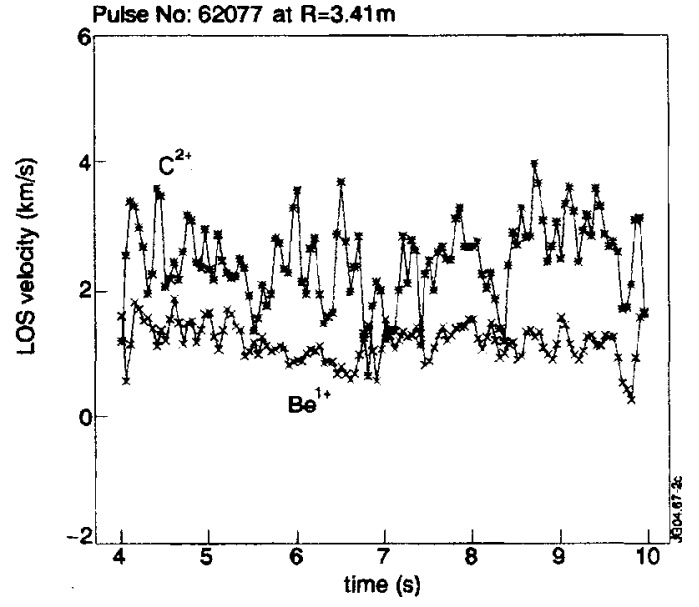

FIG. 1. Line of sight velocities of $\mathrm{Be}^{1+}$ and $\mathrm{C}^{2+}$ ions using a recent lamp calibration.

flows are very low. Using shots that directly follow the lamp calibrations, the average velocities along the LOS of these ions are measured to be less than 5 km/s, as shown in Fig. 1.

Assuming that the average line of sight velocities of these ions are negligible compared with the confined plasma $\mathrm{C}^{6+}$ ions, the central position of the $\mathrm{C}$ vi line is also defined from the locations of the measured $\mathrm{Be}$ II and $\mathrm{C}$ III lines. If a recent lamp calibration is not available, the inclusion of the second calibration method can significantly change the measured absolute values of the poloidal rotation, in some cases by more than $15 \mathrm{~km} / \mathrm{s}$, as is shown in Fig. 2. The advantage of using the second calibration method in addition to the first is that it can provide a check on a shot to shot basis for plasmas with sufficiently strong plasma line intensities. Results from both methods agree closely, when a recent lamp calibration is used.

\section{MEASUREMENTS OF THE TOROIDAL CONTRIBUTION}

Since the viewing chords of the CXRS diagnostic have a toroidal component, experiments were performed to evaluate

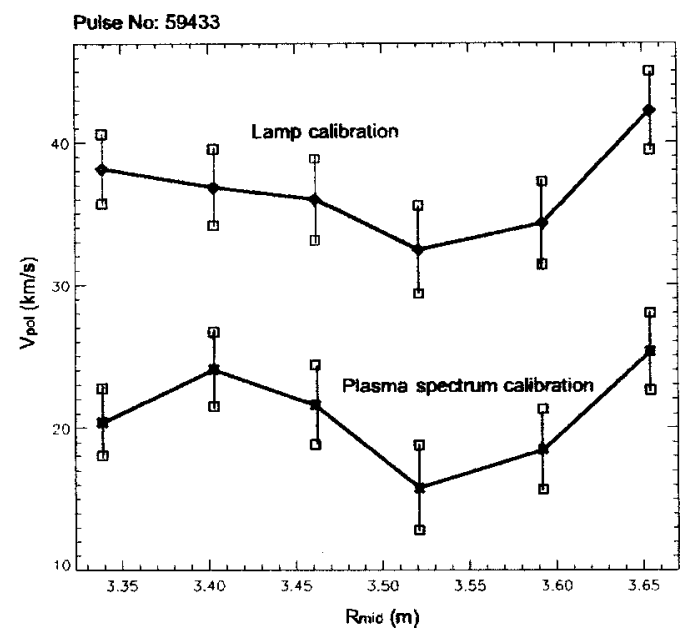

FIG. 2. Poloidal rotation velocity using both (i) a lamp calibration that was taken two weeks before the actual shot and (ii) the plasma spectrum. The influence on the profile is mainly an offset in the absolute values. Different errors are taken into account: error in the spectral line fit, in the toroidal velocity and in the calculation of the viewing geometry, i.e., the angles between the viewing chords and the toroidal and poloidal magnetic field.

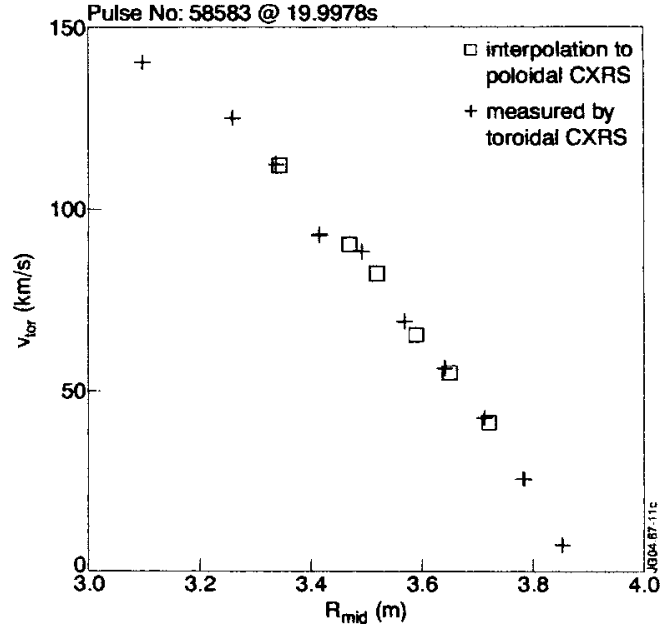

FIG. 3. The toroidal rotation velocity measured by the toroidal CXRS diagnostic and the interpolation to the position of the viewing chords of the poloidal rotation velocity $\mathrm{CX}$ diagnostic.

this contribution and to compare with the calculated values. The calculations take into account the geometry of the viewing lines, of the neutral injectors used and of the plasma magnetic configuration. During these experiments the plasma remained in $L$ mode. Experimentally we find a low and constant contribution from the poloidal component as shown in Fig. 2 of Ref. 2 . In these $L$-mode shots, the toroidal rotation velocity was increased in steps using the tangential neutral beam injections to cover as wide a range as possible. The total line of sight velocity $v_{\text {los }}$, determined from the Doppler shift of the spectral line, can be written as

$$
\nu_{\text {los }}=c \frac{\Delta \lambda}{\lambda_{0}}=\nu_{\text {tor }} \cos \phi+\nu_{\mathrm{pol}} \cos \theta,
$$

where $v_{\text {tor }}$ is the toroidal velocity at the location of the viewing chords, and $\phi$ and $\theta$ are the angles between the lines of sight and the toroidal and poloidal magnetic field components respectively. A second CXRS diagnostic on JET with a purely tangential view was used to provide interpolated measurements of the toroidal rotation velocity $v_{\text {tor }}$ at the line of sight locations, shown in Fig. 3 . In the $L$-mode phase the second term on the right hand side in Eq. (1) is small $(<5 \mathrm{~km} / \mathrm{s})$, and it remained constant during these discharges as $v_{\text {tor }}$ was increased. The contribution of the toroidal component (i.e., $\cos \phi$ ) was then derived directly from the measurements. In Fig. $4 v_{\text {los }}$ is plotted as a function of $v_{\text {tor }}$ and the straight line represents the best fit to the data, where the

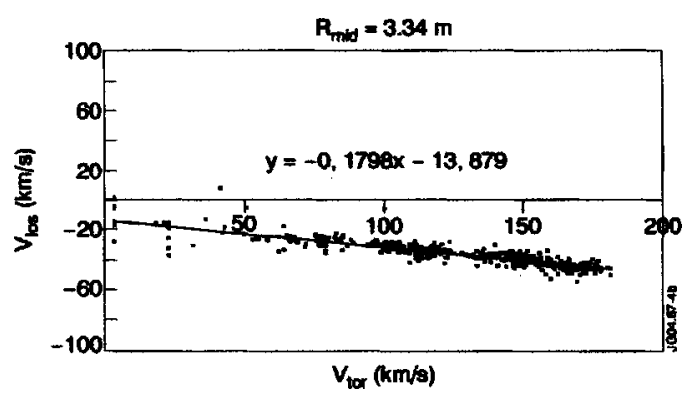

FIG. 4. Determination of the $\cos \phi$ from the measurements of $v_{\text {los }}$ and $v_{\text {tor }}$ for the fiber at radial location $R=3.34 \mathrm{~m}$. 


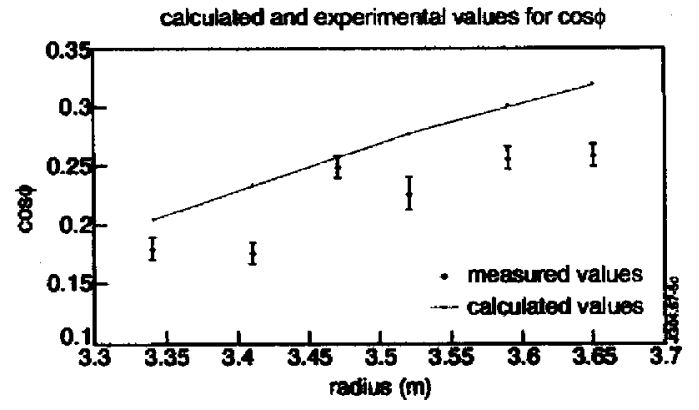

FIG. 5. Comparison of calculated and measured values for the contribution of the toroidal component to the total line of sight velocity.

slope provides an experimental value for $\cos \phi$. Figure 5 shows that the experimental values for the different viewing chords are about $15 \%$ lower than the calculations. The effect of using the experimental versus calculated angles on the measured $v_{\text {pol }}$ in the same discharge is illustrated in Fig. 6 and is found to be about $5 \mathrm{~km} / \mathrm{s}$.

\section{EXPERIMENTAL RESULTS}

Temperature corrections must also be applied for the measurement of $v_{\mathrm{pol}}$ and $T_{i}$. The dependence of the effective rate coefficient on collision energy introduces an apparent line shift ${ }^{3}$ and the gyromotion during the finite lifetime of the higher exited states introduces an additional line shift, due to the partly poloidal view of the chords. ${ }^{4}$ On JET only temperature corrections for the energy dependence of the cross sections are applied. CXRS measurements of $v_{\mathrm{pol}}$ were therefore made during a discharge with a mode-lock to evaluate if

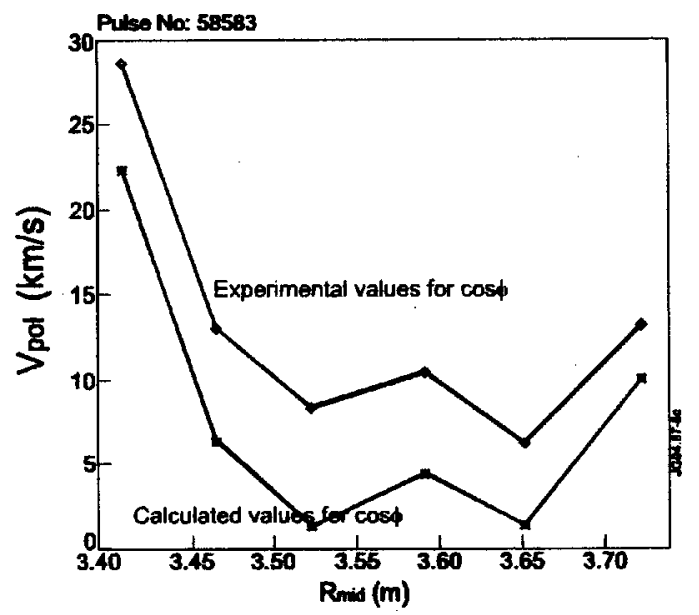

FIG. 6. Effect of the difference in $\cos \phi$ values on the poloidal rotation velocity profiles.

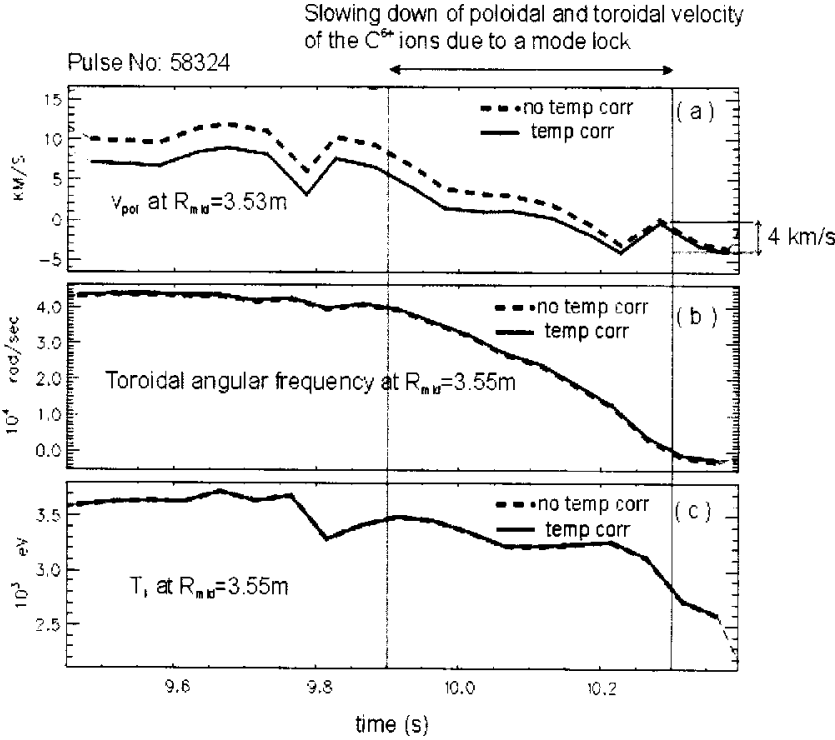

FIG. 7. (a) The time evolution of $v_{\text {pol }}$, (b) the toroidal angular frequency, and (c) the ion temperature all around the time of a mode lock.

the temperature corrections being used are sufficient. ${ }^{5}$ At the time of the mode-lock all plasma rotation reduces to zero immediately. In Fig. 7(a) it can be seen that the apparent line shift due to the temperature effects results in a difference in the values of $v_{\text {pol }}$ of about $3 \mathrm{~km} / \mathrm{s}$ for this example where $T_{i}$ is about $3 \mathrm{keV}$ [Fig. 7(c)]. Both toroidal and poloidal velocities break down at $9.9 \mathrm{~s}$, as is indicated in Figs. 7(a) and 7(b). When the toroidal velocity is stopped completely at $10.3 \mathrm{~s}$, the poloidal velocity reaches $\sim 0 \mathrm{~km} / \mathrm{s}$ as well. From Fig. 7(a) we know that possible additional apparent line shifts, due to gyro-orbit motion must be $<4 \mathrm{~km} / \mathrm{s}$. For higher ion temperatures however, the effects may become more important, and additional corrections might have to be included.

In addition, good agreement is found between the $T_{i}$ values obtained with the improved diagnostic and those from (i) the toroidal CXRS diagnostic and (ii) an x-ray crystal spectrometer at JET, observing $\mathrm{Ni}^{26+}$ ions in the plasma core.

${ }^{1}$ N. C. Hawkes, Rev. Sci. Instrum. 68, 2051 (1997).

${ }^{2}$ K. Crombe et al., Proceedings 30th EPS Conference on Controlled Fusion and Plasma Physics, St-Petersburg, Russia, 7-11 July 2003, ECA Vol. 27A, p. 1.55 .

${ }^{3}$ M. von Hellermann, P. Breger, J. Freiling, R. König, W. Mandl, A. Maas, and HP Summers, Plasma Phys. Controlled Fusion 37, 71 (1995).

${ }^{4}$ R. E. Bell and E. J. Synakowski, PPPL-3447 AIP Proceedings of the 12th Topical Conference on Atomic Procs., Reno, Nevada, 19-23 March 2000, Princeton University, Princeton, New Jersey, May 2000.

${ }^{5}$ H. Meister, A. Kallenbach, A. G. Peeters, A. Kendl, J. Hobirk, S. D. Pinches, and ASDEX Upgrade team, Nucl. Fusion 41, 1633 (2001). 